\title{
Comparing Shear Bond Strength of Two Step vs One Step B onding Agents on Ground Enamel and Dentin: An in vitro Study
}

${ }^{1}$ Darshan Deepak Shah, ${ }^{2}$ Manoj Chandak, ${ }^{3}$ Narendra Manwar, ${ }^{4}$ Shubhangi Mani, ${ }^{5}$ Ameet Mani

${ }^{6}$ Rajiv Saini, ${ }^{7}$ Rushabh Malde

\section{ABSTRACT}

Aim: To compare the shear bond strength of Tetric $\mathrm{N}$ Bond (Ivoclar Vivadent) and Single Bond Universal Adhesive (3M ESPE, MN, USA) on ground enamel and dentin.

Materials and methods: A total of 30 extracted human maxillary and mandibular molars and premolars were used for this study and divided into two groups which were treated with Tetric $\mathrm{N}$ Bond (Ivoclar Vivaden) t- and Single Bond Universal Adhesive (3M ESPE, MN, USA). The shear bond strength was tested on Universal testing machine (Instron).

Results: There was statistically significant difference in the shear bond strength among the two groups.

Conclusion: Based on the results (Tetric N Bond, Ivoclar Vivadent) had higher bond strength than Single Bond Universal Adhesive (3M ESPE, MN, USA) on ground enamel and dentin.

Keywords: Shear bond strength, Tetric $\mathrm{N}$ bond, Single bond universal adhesive.

How to cite this article: Shah DD, Chandak M, Manwar N, Mani S, Mani A, Saini R, Malde R. Comparing Shear Bond Strength of Two Step vs One Step Bonding Agents on Ground Enamel and Dentin: An in vitro Study. Int J Experiment Dent Sci 2014;3(1):1-3.

\section{Source of support: Nil}

Conflict of interest: None

\footnotetext{
${ }^{1}$ Postgraduate Student, ${ }^{2}$ Professor and Head, ${ }^{3,4}$ Professor

${ }^{5}$ Associate Professor, ${ }^{6}$ Associate Professor, ${ }^{7}$ Private Practitioner

${ }^{1-3}$ Department of Conservative Dentistry and Endodontics Sharad Pawar Dental College, Wardha, Maharashtra, India

${ }^{4}$ Department of Orthodontics, Rural Dental College, Pravara Institute of Medical Sciences, Ahmednagar, Maharashtra, India

${ }^{5}$ Department of Periodontics, Rural Dental College, Pravara Institute of Medical Sciences, Ahmednagar, Maharashtra, India

${ }^{6}$ Department of Periodontology and Oral Implantology Rural Dental College, Pravara Institute of Medical Sciences Ahmednagar, Maharashtra, India

${ }^{7}$ Private Practitioner, Mumbai, Maharashtra, India
}

Corresponding Author: Darshan Deepak Shah, Postgraduate Student, Department of Conservative Dentistry and Endodontics Sharad, Pawar Dental College, Wardha, Maharashtra, India Phone: 9860078925, e-mail: darshanshah2609@gmail.com

\section{INTRODUCTION}

In 1955, Buonocore laid the foundation of modern adhesive dentistry, when he reported that acids could be used to alter the surface of enamel to render it more receptive to adhesion. ${ }^{1}$ Over the past 2 decades, significant improvements have been done in the field of dentin adhesives and restorative dentistry. Fusayama et al in 1979, proved that good dentin adhesion can be achieved in vivo by acid etching the dentin before an unfilled resin was placed on the dentin surface. Dentin bonding agents, used during the early $90 \mathrm{~s}$, are still in use and are often referred to as a fourth generation of dentin adhesives. Dentist thought that 4th generation of adhesives were complex and time consuming and demanded simpler solutions. The first simplification was the 5 th generation of bonding agents, systems in which primer and the adhesive were mixed together and supplied as a simple system. After the development of 5th generation, the demand for simpler systems increased as a result of which two such systems evolved: one consisting of an acid primer and a bonding agent resin referred to as a 6 th generation adhesive, and another in which the etchant, primer, and adhesive are combined in a single delivery system marked as seventh generation of adhesive systems. Various studies, evaluating shear bond strength of self-etching adhesive have shown inconclusive results on permanent dentition. The advantages of the self-etching system include complete infiltration of the bonding agent into the demineralized dentin and a reduce number of clinical procedural steps. One of the problems faced in adhesive dentistry is resin-dentin bond degradation by water and vapor over a period of time. Bonding to enamel remains the simplest and most reliable of all adhesive procedures. ${ }^{2}$ Bonding to the dentin is where the clinician faces difficulty mainly due to the contents of the dentin, i.e. $45 \%$ inorganic and the rest being organic and water. Lower bond strength to dentin occurs as a result of numerous factors. ${ }^{3}$

Dentin contains less mineralized tooth structure and more water than enamel.

Outward pressure from pulp reduce the stability of the bond between the composite resin and dentin.

The presence of smear layer makes the wetting of dentin very difficult. 
To overcome these problems, dental adhesive systems have evolved through several generations with changes in mechanisms, chemistry, number of bottles, application techniques and clinical effectiveness. Laboratory in vitro test play an important role in providing the necessary information regarding the efficacy of new products in a short period of time and at a lesser cost, whereas clinical evaluations would provide information only after along period of use. -6 $^{4-6}$

The present study is performed to assess the shear bond strength on ground enamel and dentin of two dentin bonding agent (Tetric N Bond, Ivoclar Vivadent and Single Bond Universal Adhesive, 3M ESPE, MN, USA) in conjunction with composite (Filtek Z 350).

\section{MATERIALS AND METHODS}

A total of 30 freshly extracted molars and premolars with unattrited, intact crowns, free from decay and fractures, were collected. The teeth were scaled with an ultrasonic scaler to remove tissue tags, plaque and calculus, and they were polished with pumice and stored in saline until further use. The teeth were sectioned into ground enamel and dentin. The specimens were grinded against the 600- sand grit paper mounted on a wheel to obtain a flat enamel and dentin test surface. Each specimen was wrapped in gauze moistened with saline and stored in a closed container at room temperature until the adhesive and restorative materials were applied. They were randomly divided into 2 groups of 15 teeth each.

Group I: A total of 15 teeth were treated according to the manufacturer's instructions with seventh generation singlestep self-etch adhesive (Single Bond Universal Adhesive, 3M ESPE, MN, USA) for the adhesion of composite resin on its enamel and dentin surface.

Group II: A total of 15 teeth were treated according to the manufacturer's instructions with 6th generation, i.e. two step adhesive (Tetric N Bond, Ivoclar Vivadent) for the adhesion of composite resin on its enamel and dentin surface.

Samples were mounted on an acrylic resin block having dimensions of $3 \times 3 \mathrm{~cm}$. Group I was bonded using Single Bond Universal System (seventh generation), while group II was bonded with Tetric N Bond, Ivoclar Vivadent (sixth generation). Following the application of adhesive, composite resin cylindrical block measuring $2 \mathrm{~mm}$ in diameter and height of $6 \mathrm{~mm}$, in a two-layer increment technique. Each

Table 1: Descriptive measurements of shear bond strength

\begin{tabular}{lllll}
\hline Groups & Samples & \multicolumn{3}{c}{ Shear bond strength } \\
\cline { 2 - 5 } & & Range & Mean & $S D$ \\
\hline I & 15 & $2.75-7.96$ & 4.60 & 1.60 \\
II & 15 & $4.33-6.72$ & 5.38 & 0.79 \\
\hline \multicolumn{3}{l}{ Unpaired t-test, $\mathrm{p}<0.05$, difference is significant }
\end{tabular}

layer was light-cure for 20 seconds with a light-curing unit (Rotex, Germany) vertically for each increment. For each specimen, the curing tip was placed as closely as possible to the composite. After the composite built-up the teeth were placed in the incubator at $37^{\circ} \mathrm{C}$ for 24 hours. The teeth were thermocycled in distilled water 100 times, between $5^{\circ} \mathrm{C}$ and $55^{\circ} \mathrm{C}$, with 5 seconds transfer time for each and 30 seconds dwell time. The shear bond strength tests were measured using the Universal Testing Machine at a cross head speed of $1 \mathrm{~mm} /$ minute.

The force required to separate the composite material from the tooth surface was registered in Newtons, and converted into MegaPascals (MPa) as a ratio of Newtons to surface area of the bonded surface $\left(\mathrm{MPa}=\mathrm{N} / \mathrm{m}^{2}\right)$. The obtained results were statistically analyzed.

\section{RESULTS}

The quantitative data were presented as mean, standard deviation, and range value. Unpaired t-test was used for comparison.

The mean bond strength values for both groups I and II are shown in Table 1. The bond strength value were obtained in KiloNewton and converted to MPa.

The mean bond strength value for group I was 4.60 \pm 1.60 and for group II was $5.38 \pm 0.79$. The p-value for comparison between groups I and II was 0.0125 , which was less than 0.05 , indicating a statistically significant difference between the two groups.

This indicated that the 6th generation adhesive was more effective on the round enamel and dentin surface as compared to the 7 th generation adhesive.

\section{DISCUSSION}

Test adhesives are screened by bond, strength, test. Wellsealed and long-lasting restorations need high shear bond strength for adhesion of resin materials to enamel, especially to dentin.

It is important to focus on simplifying procedures in adhesive technology. What is needed is reducing technique sensitivity. Additional chemical reaction with the tooth substance is also needed.

The shear bond strength of a sixth generation bonding agent (Tetric N Bond, Ivoclar Vivadent) vs seventh generation bonding agent (Single Bond Universal Adhesive, 3M ESPE, MN, USA) is compared in present study. There are reasons for the superior performance of two-step self- etch systems:

1. The solvent present has low concentration. ${ }^{7}$

2. The hydrophylicity is low. ${ }^{7}$

3. Polymerization is to a greater degree. ${ }^{7}$ 
4. The underlined dentin undergoes limited etching and demineralization over a longer period of time. ${ }^{8}$

5. Ethanol is present in Tetra N Bond (group 2) and Acetone is Present in (Single Bond Universal Adhesive (group 1). ${ }^{9}$

On the basis of $\mathrm{pH}$ there are two types of self-etch adhesives; Strong ( $\mathrm{pH}$ less than 1) and Mild ( $\mathrm{pH}$ more than 1). Deep mineralization equivalent to phosphoric acid etching is caused by high acidity for strong self-etch adhesives. ${ }^{10} \mathrm{~A}$ substantial number of hydroxyapatite crystals remain within the hybrid layer when the mild self-etch adhesive dissolves the dentin surface only partially. ${ }^{10}$

The functional monomers are delivered into the hybrid layer by the organic solvents, ethanol and acetone which act as carriers and water chasers. Acetone is more volatile than ethanol because acetone has vapor pressure $200 \mathrm{~mm} \mathrm{Hg}$ at $25^{\circ} \mathrm{C}$ where else Ethanol has $54.1 \mathrm{~mm} \mathrm{Hg}{ }^{11}$

Etching, priming and bonding both hydrophilic and hydrophobic monomers are combined in one step adhesives and are blended with a relatively high concentration of solvent to keep them in solution. To enable self-etching activity, water is also essential as an ionization medium.

Serious limitations of all in one adhesive are: continued demineralization of the adjacent dentin structure in the tubules and incomplete polymerization. ${ }^{11}$

Two bottles are involved in two step, etching and priming and then bonding. To allow deeper penetration, the all in one adhesive needs to be acidic and the formulations have become more hydrophilic. ${ }^{8}$

The water content increases when the adhesive penetrates the wet dentinal tubules deeply. It has been shown by studies that this water acts as a major interfering factor in polymerization. It leads to unpolymerized acidic and aggressive monomers and continue etching the dentin, creating detrimental impact on the bond.

Harsh conditions of oral environment, such as intraoral temperature, moisture contact, fatigue of bond due to tooth flexure, and bacterial enzymes also lead to low bond strength values and failure of resin based adhesives in vivo.

On the basis of all these studies, superior in vitro performance was shown by two-step etch systems in terms of shear bond strength compared to one step self-etch system. ${ }^{12}$

For evaluating the efficacy of bonding materials, bond strength studies are quite rough. In vitro bond strength to dentin is influenced by several factors, such as the type and age of the teeth, the degree of dentin mineralization, the bond of dentin surface, the type of bond strength test, the storage media, and the environmental surface humidity.

On the basis of this study, higher shear bond strength was exhibited by then contacts and higher bond strength was exhibited by permanent teeth compared to primary teeth, though it is insignificant.

The less pronounced enamel etching pattern obtained with the self-etch adhesive causes lower adhesion strength of the Single Bond Universal Adhesive to intact enamel surfaces, due to in adequate $\mathrm{pH}$ of the primer.

Intact enamel walls less accessible to self-etch adhesives, as it was hypermineralized and may contain more fluoride than instrumented enamel.

\section{CONCLUSION}

Within the limitation of this in vitro study the conclusions drawn are:

The shear bond strength of Tetric $\mathrm{N}$ Bond is significantly higher compared to that of Single Bond Universal Adhesive on ground enamel and dentin. However, these results have to be substantiated with further in vitro and long-term clinical trials and studies.

\section{REFERENCES}

1. Swift EJ. Enamel/Dentin adhesives: Pennsylvania academic of general dentistry-Dr. Swift article 2002. 1-14 p.

2. Kakar M. The chemistry of bonding; pathways to perfection. 2002. 1-4 p.

3. Pashley DH. The evolution of dentin bonding from no-etch to total etch to self-etch, adhesive technology solutions, premier Issue; Kuraray 2002. p. 1-8.

4. Soderholm KJ, Guelmann M, Bimstein E. Shear bond strength of one 4th and two 7 th generation bonding agents when used by operators with different bonding experience. J Adhesive Dent 2005;7(1);57-64.

5. Nakabayashi N, Kojima K, Masuhara E. The promotion of adhesion by the infiltration of monomers into tooth substrates. J Biomed Marter Res 1982:16(3):265-273.

6. Tay FR, Pashley DH. Dental adhesives of the future. J Adhes Dent 2002;4(2):94-103.

7. Van LanduytK1, De Munck J, Snauwaert J, Countinho E, YoshidaY, Kall S, Poitevin A, Yoshida Y, Inoue S, Peumans M, et al. Monomer-solvent phase separation in one step self-etch adhesives. J Dent Res 2005;84(2):183-188.

8. Wang Y, Spencer P. Continuing etching of an all in one adhesive in wet dentin tubules. J Dent Res 2005;84(4):350-354.

9. De Munck J, Van Landuyt K, Peumans M, Poitevin A, Lambrechts P, Bream M, Van Meerbeek B. A critical review of the durability of adhesion to tooth tissue: methods and results. J Dent Res 2004;84(2):118-132.

10. Koshiro K, Inoue S, Sano H, De Munck J, Van Meerbeek B. In vivo degradation of resin dentin bonds produced by a self-etch and an etch-and-rinse adhesive. Eur J Oral Sci 2005;113(4):341-348.

11. Cheng JT, Itoh K, Kusunoki M, Hasegawa T, Wakumoto S, Hisamitsu H. Effect of dentine conditioners on bonding efficacy of one bottle adhesives. J Oral Rehabil 2005;32(1):28-33.

12. Burrow MF, Harada N, Kitasako Y, Nikaido T, Tagami J. Seven years dentin bond strengths of a total and self-etch system. Eur J Oral Sci 2005;113(3):265-270. 